Proceedings of the 2010 Winter Simulation Conference

B. Johansson, S. Jain, J. Montoya-Torres, J. Hugan, and E. Yücesan, eds.

\title{
A MULTIPURPOSE SIMULATION PLATFORM FOR DECISION-MAKING IN CONSTRUCTION MANAGEMENT
}

\author{
Corey Tebo \\ Department of Computer Science \\ Michigan Tech. \\ Houghton, MI 49931, U.S.A.
}

\author{
Amlan Mukherjee \\ Department of Civil and Environmental Engineering \\ Michigan Tech. \\ Houghton, MI 49931, U.S.A.
}

\author{
Nilufer Onder \\ Department of Computer Science \\ Michigan Tech. \\ Houghton, MI 49931, U.S.A.
}

\begin{abstract}
Research in general purpose and special purpose simulation platforms typically treat model development, experimentation, validation and deployment of simulations as distinct phases. Direct involvement of decision-makers is usually limited to the validation phase, even though their participation significantly improves the effectiveness and applicability of models. Unfortunately, the complexity and sophistication involved in the model development and experimentation phases deters their participation. This also makes the validation problem particularly challenging, and hinders the credibility and successful deployment of such models. In addressing this problem, we introduce an interactive simulation platform called Interactive Construction Decision Making Aid (ICDMA), that integrates decision-maker participation into the model development, simulation deployment, experimentation and validation phases. Effectively it separates the complexities of programming the model and the model development process, thus encouraging the participation of domain experts. We illustrate the usefulness of this platform.
\end{abstract}

\section{INTRODUCTION}

Simulations are arguably one of the most crucial tools in construction engineering and management. These tools simulate construction project scenarios in keeping with the rules that govern the construction management (CM) domain in general, and the project being simulated in particular. The effective use of simulations in construction decision making requires the adoption of formal steps including model definition, data synthesis, verification and validation, and empirical work. These steps are revisited until the experimental results are deemed satisfactory for decision making.

During the revision process from model definition to validation, researchers and stake-holders become frequent users of simulation systems. Pritsker (1992) defines four types of simulation users. The hands-off type users rely on the expertise of simulation developers and use the results provided by them. The requester type users provide guidance and interpret outputs, but do not run simulations directly. The hands-on type users run simulations but might not have the background to change or create models. Finally, renaissance type users can make decisions on simulation models, database schemas, and other application programs. In applications where the simulator can be viewed as a black box which takes inputs and produces outputs, simulator platforms that support the first three types of users is effective. However, when a simulator serves different types of tasks ranging from decision making support to experimental validation, the needs of the renaissance type of user must be met.

In this paper, we present the Java programming language implementation of an interactive simulation platform called Interactive Construction Decision Making Aid (ICDMA). The design of ICDMA emphasizes representing and reasoning with the constraints that govern the planning and execution of a construction project. These constraints as well as the construction activities and stochastic temporal events are represented in a structure called the TONAE (TempOral Network with Activities and Events). The TONAE structure and the associated algorithms are explained in the next section. Next, we show the ICDMA architecture. We then discuss two example platforms where ICDMA is deployed. We conclude with a summary and future directions.

\section{TEMPORAL NETWORK WITH ACTIVITIES AND EVENTS (TONAE)}

At the heart of any system lies the ability to mathematically represent domain knowledge and formally reason about changes in the system. The reasoning is dependent on knowledge about the construction management domain. For 
example, in order to lower productivity in the event of bad weather, it is necessary for the reasoning entity to be aware of the dependence between bad weather and productivity. When propagating a delay in a schedule the reasoning entity can automatically update the status of variables, while using domain knowledge to infer future events and project outcomes. Such knowledge may be project specific - such as space constraints in urban projects, or they may generally apply to the construction management domain - such as, reduced productivity due to labor overtime. It is critical to represent such constraint information explicitly to allow reasoning that can track the impact of constraint violations through out the project.

For the construction environment a temporal representation is used. It is a combination of variables based on the reasonable abstraction of construction schedules as temporal commitments. Using the as-planned schedule as the baseline commitment, variations can be captured and formally expressed as a set of altered commitments in the as-built schedule. The comparison of these commitments - or keeping track of change - as the project temporally evolves provides a useful foundation for reasoning about the construction management process (Rojas and Mukherjee 2005b).

The implementation of the TONAE module consists of a temporal data structure of project activities and the algorithms that update the data structure. The data structure contains information about the project activities, external events that affect the project, and the current status of the project as shown in Figure 1a. Each activity $A_{i}$ has a beginning point $\left(A_{i, B}\right)$ and an end point $\left(A_{i, E}\right)$ that are shown as circles. The links between the beginning point and the end point of the same activity initially show the as-planned duration of the activity. For example, activities $A_{1}$ and $A_{4}$ have as-planned durations of one unit. The links between the beginning and end points of different activities show the constraints between activities. For example, the links labeled 0 from $A_{1, E}$ show that the activities $A_{2}$ and $A_{4}$ are scheduled to start right after activity $A_{1}$ in order not to keep the labor crews idle. The rectangular boxes show the external events that affect the project. In Figure 1a, a snow event with duration one is shown between $E_{1, B}$ and $E_{1, E}$. This snow event starts at time $T_{3}$ and is expected to last for one time unit. During this time, it will affect all the activities as specified by the rules regarding the project environment. For example, bolting activities will be slowed down because the workers will need to wear gloves during cold weather. The triangular box shows the current time point in the simulation. The project in the example is at time point $T_{3}$. The link labeled 1 from $A_{2, B}$ to $Y$ shows that activity $A_{2}$ is currently running and, it started one time unit ago, at time $T_{3}$. The link also labeled 1 from $Y$ to $A_{2, E}$ shows that activity $A_{2}$ is expected to finish within one time unit.

Event $E_{1}$ is a snow event that takes one time unit. If Activity $A_{2}$ is an activity that is affected by snow, its finish time will be pushed from time $T_{4}$ to the extent of the effect. The extent of the effect is recorded in the database, e.g., productivity decreases by $25 \%$.

The TONAE also keeps information about material and resource allocations to each activity. The underlying temporal representation differentiates it from traditional discrete event simulations - instead of advancing the simulated time from one discrete event to the next, it advances time from one time point to the next contiguous time point. The simulation is not dictated by a calendar of specific events, but instead it is an emulation of a passage through time. If no events take place during the course of the project, the data structure is advanced by one time unit at a time as instructed by a driver module which is explained in the next section. Hence, at each time point $T$ the simulation is ascribed a state $\mathbf{S}_{T}$ which is expressed by a constraint network of activities and events called the TONAE (Anderson, Mukherjee, and Onder 2009).

The two main algorithms in the TONAE module are Traverse and Query. Traverse takes the project status at time $T_{i}$ and advances it to the next time point $T_{i+1}$ by randomly generating the events that take place at $T_{i}$, computing the effects of these events on the activities, and calculating the updated durations and costs. Query uses an algorithm similar to Traverse to obtain the project status without modifying the data structures. The Traverse algorithm is used to advance the time in a simulator setting. The querying algorithm is used to develop risk distributions at the end of each time point. It queries the combinatorial future space of project outcomes using a Monte-Carlo sampling method at the end of each time point and classifies the results by impact and probability (Anderson, Mukherjee, and Onder 2009).

TONAEs advance the existing representation in three ways: (i) They can represent intervals as well as constraints between individual time points defining such intervals, (ii) They provide an integrated approach to express all the constraints, activities and events using a temporal network and (iii) They provide the ability to develop general algorithms that could be used to traverse and query the network to aid decision-making and planning. From a methodological perspective, the TONAE representation provides the ability to reason about the past, current, and future status of the simulated construction project. This creates opportunities to explore the sensitivity of project risk and uncertainty to relationships between simulation variables, and alternative decision strategies.

\section{INTERACTIVE CONSTRUCTION DECISON MAKING AID (ICDMA)}

ICDMA is a continuous time advance simulation system that uses TONAE's ability to provide a rich state based representation of construction information and constraints. Figure $1 \mathrm{~b}$ shows the main modules of the system. The TONAE and the Driver together comprise the simulator as explained below. The Graphical User Interface (GUI) to the driver allows users interact with the simulator to change state variables such as material allocations, or to view the simulator results. The project description is stored in a database which can be accessed through a separate GUI. All 


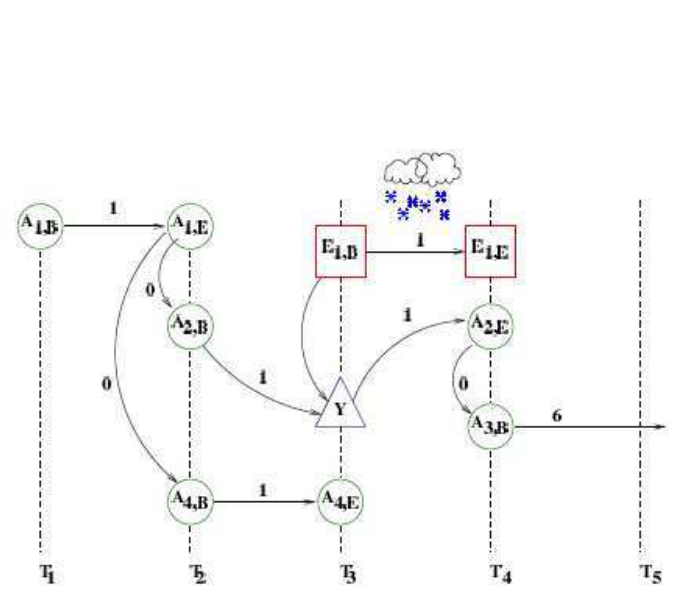

(a) The TONAE data structure

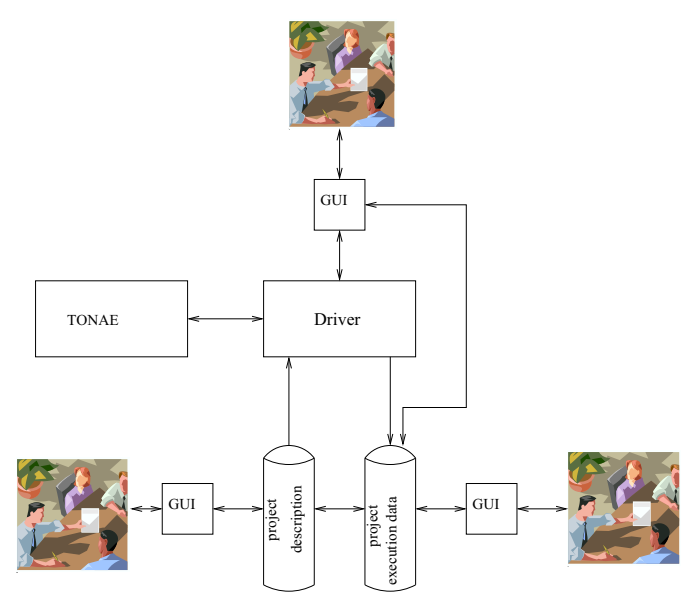

(b) The modules of the ICDMA framework.

Figure 1: Frameworks

the data collected during the simulation run is stored in a second database. This database can be analyzed separately or through the information it feeds to the Driver's GUI.

At each simulated time point $T$ (the time unit depends on the granularity preferred by the user), the Driver takes the inputs from the planner or decision maker who has the ability to directly control the work flow in the simulated project. Using this input and its internal state representation the TONAE transitions to a new state $\mathbf{S}_{T+1}$. This implies that each time point in the simulation is effectively a control point allowing decision-makers to experiment with different strategies and examine outcomes associated with alternative decisions. The simulation is capable of formally representing decisions at each time point using a set of decision variables $-\mathbf{C}_{T}$. Hence the rate of change of the system can be expressed mathematically by the equation:

$$
\mathbf{S}_{T+1}=\left[\mathbf{C}_{T}+\mathbf{R}\right] \bullet \mathbf{S}_{T}
$$

where $\mathbf{R}$ is the impact of random events - such as bad weather, change orders, create on the progress of the project simulation. The - operation symbolizes the impact of the decisions and random variables on the current state of the system $\left(\mathbf{S}_{T}\right)$ to create the new state of the system $\mathbf{S}_{T+1}$. Currently the simulation can be used to represent the impact of external events and decisions on the evolution of the simulation from state to state. Unlimited control points in the simulation also provides an interactive platform capable of providing project specific, context rich strategy analysis and contingency plans. Alternative project management strategies can be compared and their impacts on eventual project outcome can be studied in the simulated project environment by statistically analyzing this stored simulation data to infer and compare the relationships symbolized by the • operation for each strategy.

Before any further discussion of how ICDMA can be applied to further decision-making research, it is important to understand how it can be set-up for specific construction projects, through the user interface intended for simulation users.

\subsection{ICDMA Set-up}

The case study involves the construction of a four-story structural steel framed office building that was built in real life. It was implemented in ICDMA (Watkins, Mukherjee, and Onder 2008) as an example based on the documentation in Daccarett and Mrozowski (1999). The building has four stories, has 80,000 square feet of built area, weighs approximately 400 tons of structural steel or about 10 pounds per square foot. Fabrication and erection cost $\$ 9$ per square foot. A total of 964 pre-fabricated structural steel members were used in the construction. The standard bay size in the building is 30 feet by 30 feet and there are 3 bays along the width and 7 bays along the length of the building.

During the planning stages the steel frame was divided into 6 sequences, each representing the order in which a zone or section of the frame is erected. The project was planned so that the construction operation involved in the sequences could be completed in parallel. Each sequence consisted of a hoisting activity, a bolting and connections activity, and a decking activity. Hence, while the erection crew was hoisting sequence 2 the decking crew could place the metal deck on the sequence 1 that had already been completed. This deck on sequence 1 in turn provided a work platform when the crew were hoisting sequence 3 above sequence 1 . This reduced the fall distances and helped in satisfying work-site safety regulations because fall distances greater than 25 feet or two floors require separate fall 


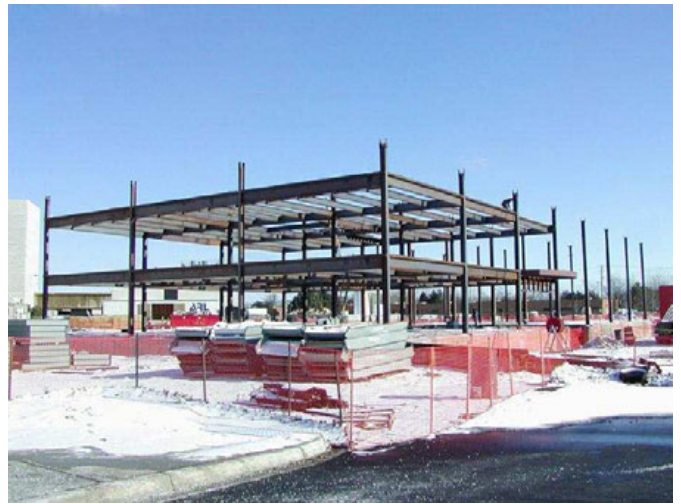

(a) Case study: after completion of sequence 3

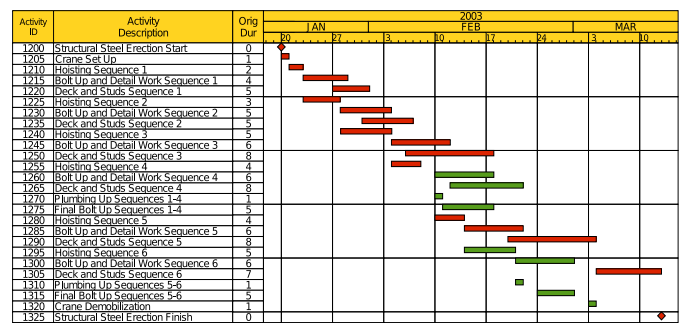

(b) Case study: project schedule.

Figure 2: Case study

protection on site (OSHA 1996). The work involved in each sequence is very similar, and the project can be considered as a combination of similar repetitive modules.

Figure 2a shows the completed hoisting activity for sequence 3. Sequences 3 and 4 consist of 365 pieces of steel while sequences 1 and 2 consisted of 166 pieces of steel. This is a hoisting rate of about 36-40 pieces a week. Seven people formed the erection crew used for this project. The crew consisted of one foreman, two iron workers hooking steel, two iron workers connecting steel, a crane operator, and an oiler. In order for the crews to be completely utilized it was important to minimize crew idle time. This meant that the hoisting crew would move from hoisting sequence to sequence with minimum time in between and would be in pursuit by the bolting and connection and decking crews. The primary information sources used to build the simulation from this case study are the documented schedule information and the documented plans and project site photographs, some of which have been illustrated in Figures $2 \mathrm{a}$ and $2 \mathrm{~b}$.

The governing constraints are formalized as follows:

- Constraint 1: In order to maintain work-flow and efficiently utilize equipment lag time between hoisting activities for any two consecutive sequences should be 0

- Constraint 2: In order to maintain labor-flow and efficiently manage crew on site, the lag time between bolting and connection activities for any two consecutive sequences should be 0

- Constraint 3: In order to ensure safety and maintain appropriate fall distance during the hoisting operations, the decking operation for a sequence should start before the hoisting operation for the sequence immediately overhead.

The simulation developer can login to the web interface and then add a new project as shown in Figure ??. Once a project has been added, the following can be done through the user interfaces:

- Adding activities to the project: These are activities that define the schedule. Hoisting, bolting and decking activities in Figure 3a, illustrate the front page of the interface with a listing of all the activities that have been added and the relevant Rules tables showing the information defining independent events.

- Adding activity specific information: For each activity constraint information, material and labor resources can be added and viewed directly by selecting to view them. The interface is shown in Figure $3 b$.

- Managing resources: The interface in Figure $3 \mathrm{c}$ is used to manage and add any new labor crews, setting up when necessary new crew compositions.

- Event management: New events can be added and existing events can be edited by defining pre and post conditions that trigger them, the probabilities of the events and consequences through the interface shown in Figure 3d.

ICDMA is a system intended to aid managers, scholars, and students in understanding the state space of a construction management plan in the context of well-defined uncertainties. The inputs described in this section set up the context information for a specific construction project that is being studied. The emulation engine emulates the project run in ICDMA as per the activity, constraint and resource information provided through the web interface. The simulation engine simulates events and disruptions as per the event definitions and probabilities provided through the web interface. 


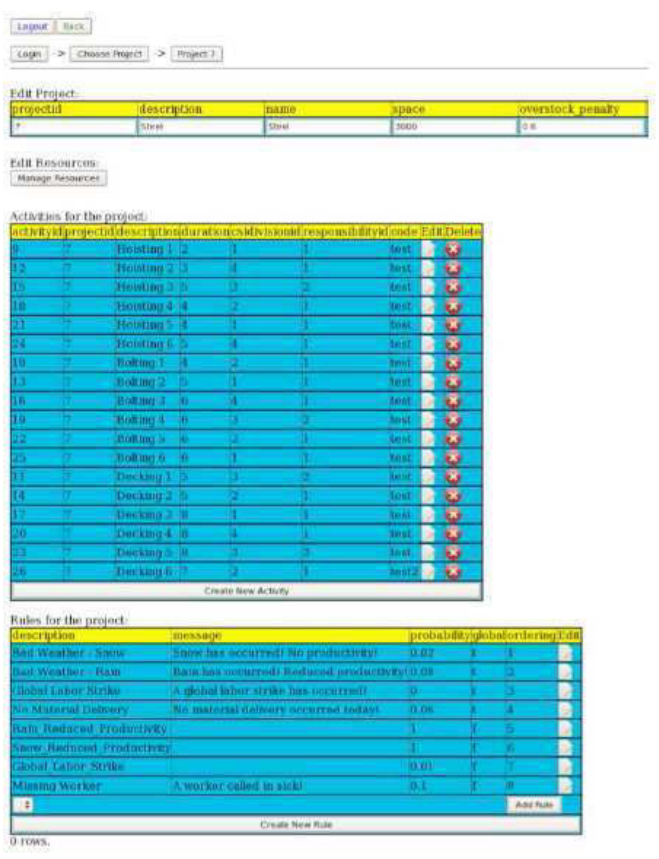

(a) Defining activities for the project in database

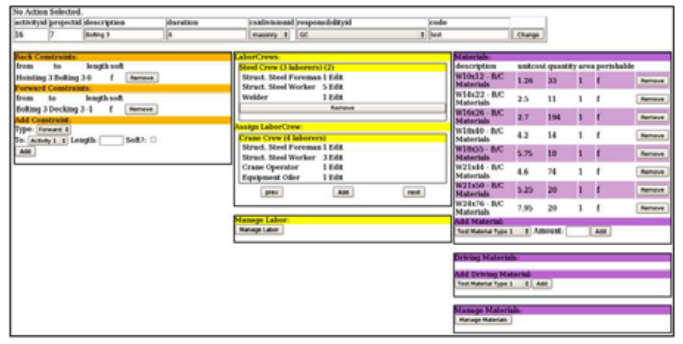

(b) Assigning resources and constraints to bolting 5 activity in database

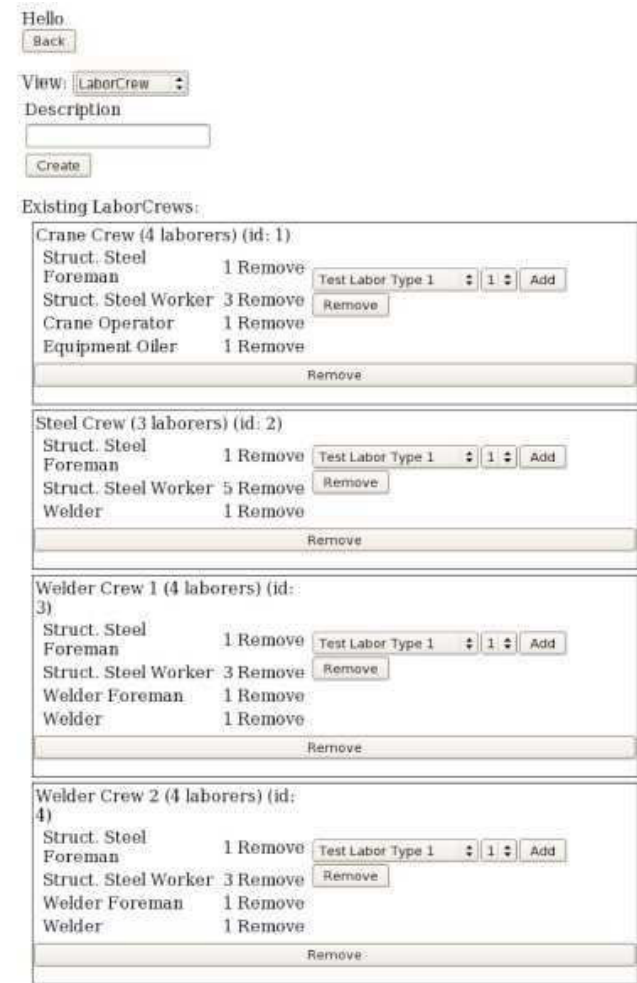

(c) Managing labor crews

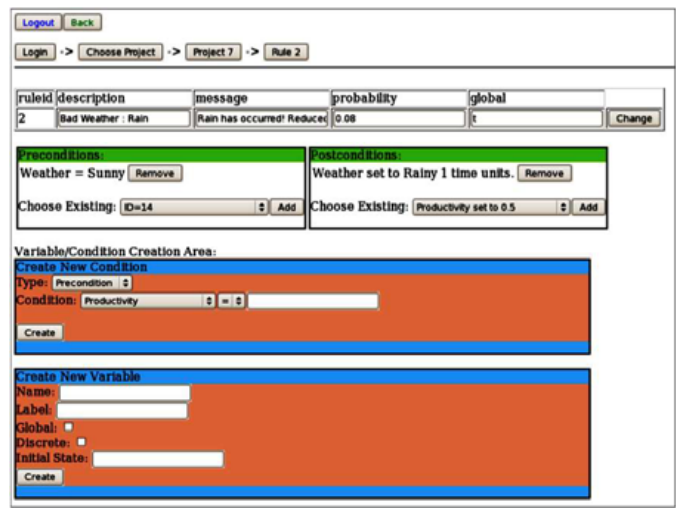

(d) Event setup in database

Figure 3: Set-up interface

\section{ICDMA APPLICATIONS}

In this section we discuss two separate applications of the ICDMA framework, one that was implemented as part of decision-making research at Michigan Tech, and the other that was implemented as an interactive simulation for construction education. Both these systems were developed by separate teams and are effectively software interfaces that implement the ICDMA framework, using the representational power of the TONAE structure. 


\subsection{Iterative Decision-Making Platform}

The first application of ICDMA is as an experimental test bed for evaluating alternative strategies and supporting an iterative decision-making process for project managers and simulation developers. The simulator developer designs the platform and the project manager uses it to interactively experiment with alternative strategies: iteratively generating new strategies and experimenting with them to refine the final set of management strategies. The decision-makers define the project or scenario to be studied and through a web-based interface that populates a back-end database with project specific information regarding constraints, event probabilities and conditions triggering events and existing after their occurrence. This information is used by the emulator and the simulator to automatically generate the TONAE and the deploy the simulation.

In the second step the simulation is run multiple times allowing interactive testing of alternative decision-making strategies. The emulator allows exploration of the project on a day to day basis. The simulator generates external disruptive events such as specified by the decision-maker. The emulator also updates the state of the simulated project at the end of each simulated time step and cascades the impacts of each disruptive event and subsequent decisions through the TONAE. ICDMA also provides feedback to the decision-makers regarding the as-planned and as-built states of the project. The following data from multiple simulation runs are archived in a back-end database:

- State information at the end of each time point $\left(\mathbf{S}_{T}, \mathbf{S}_{T+1} \ldots\right)$

- The list of external events $(\mathbf{R})$ and the times in the project when they occur

- All decisions made by the decision-maker in terms of resource allocated and schedule changes $\left(\mathbf{C}_{T}\right)$

- Outcomes from the querying algorithm at the end of each time point $T$.

An illustration of the interactive interface can be seen in Figure 4.1.

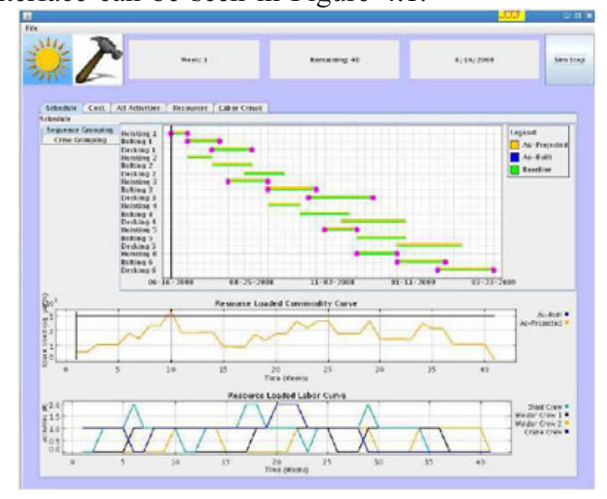

Figure 4: Interface for ICDMA - developed at Michigan Tech

The data is analyzed through statistical clustering methods to provide decision-makers the ability to compare and contrast the outcomes of alternative strategies. This information loops back into the strategy generation phase allowing them to generate and test hybrid strategies. Such a platform is critical to planning and decision-making for complex construction projects in which the occurrence of the same external event - such as a bad weather event - at different times in the schedule can have very different project outcomes. The research explores:

- Metric definition and data collection: How the querying algorithm can be used to collect risk assessment metrics for a comparative strategies

- Statistical analysis: How statistical approaches can be used to analyze and cluster outcomes from alternative strategies

These two steps are at the heart of applying ICDMA as an experimental test bed to study construction management decision-making. They have been developed in a separate paper presented in this same track.

\subsection{Application for Construction Education}

The second application, the Virtual Coach, utilizes the ability of interactive simulations to provide situational awareness. It is used in construction education to provide students clinical exercises in decision-making within interactive dynamic virtual environments. There is evidence in education literature that interactive virtual environment allow students to explore and construct knowledge through authentic experience, thus enhancing their ability to learn by connecting ideas that are otherwise provided through fragmented classroom discourse (Rojas and Mukherjee 2005a). 
Given the emphasis on situation awareness, this implementation emphasized the user interface development providing the users access to significant information views while the construction project is being simulated. An intuitive, well developed interface (Figure 4.2), was implemented using a complex real life construction project. Collaborators of the authors have developed a simulation of a real life construction project in collaboration with construction companies (Mukherjee, Onder, and Rojas 2009).

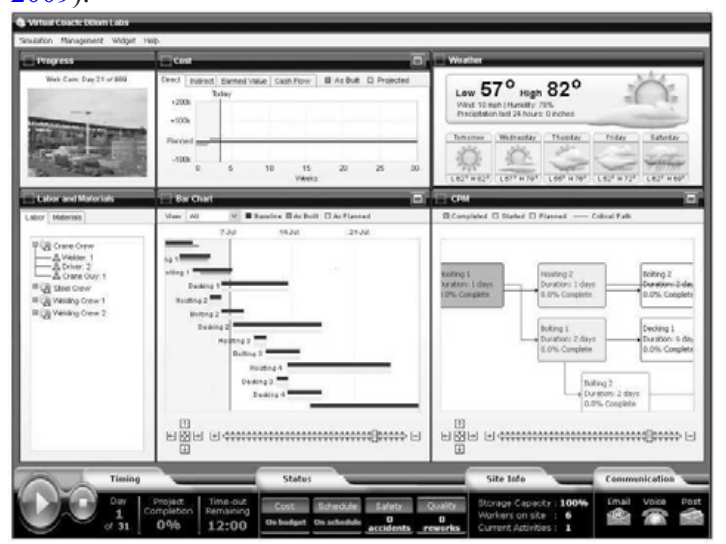

Figure 5: Interface for educational purposes - developed at University of Washington

\subsection{Validation Considerations}

Fundamentally, the software system discussed in this paper is a system that accounts for work done on each simulated day for a construction project using information from a specific construction project (Rojas and Mukherjee 2003). TONAE is a data structure that stores project and domain constraints within a temporal network. The Traverse algorithm merely advances the simulation from one time point to the next by updating the TONAE data structure using information that has been provided by the user. The Query algorithm is a Monte Carlo simulation of all the future variations that TONAE can traverse given statistical distributions of unexpected events. The outcomes from these simulation exercises are as valid as the inputs to the system. It is important to recognize that unlike discrete event simulations, the purpose of ICDMA is not to predict the behavior of a particular project metric - rather it is to support the decision-making process through a deliberative consensus building process.

Decision-making may be considered as an iterative problem solving method that converts certain and uncertain information about a domain into effective actions or decisions. It entails an iterative strategy selection loop involving (i) generation of strategies, (ii) experimenting with the outcomes associated with each strategy using suitable simulation platform, and (iii) analysis and comparison of alternative outcomes and their behaviors. This is a departure from the formulation of decision-making problems as an optimal choice between options defined by aggregate project metrics such as cost and duration. Instead, it involves the decision-maker in actively investigating the domain by generating and testing each option. The goal of such a decision-making process is to further the comprehension of how the domain and the specific problem at hand behaves, rather than predicting the exact outcome of specific metrics.

The most critical tool underlying the study of decision-making is the model of the problem being investigated. Effective communication of the model to stakeholders and decision-makers, in order to gain their trust and credibility lies at the heart of validating the model (Martinez 2009). The model itself is defined as a useful abstraction of a system or operation that allows experimentation and analysis. Traditionally, construction models are considered to be a product that is created by developers, before it is independently validated through project specific implementation by decision-makers and domain experts. There are no platforms that consider modeling as a consensus building process, allowing decision-makers and stakeholders to establish the model through a deliberative process. The need for such a platform is particularly important when considering alternative strategic project management approaches. It bridges the disjunction between the the model development and validation phase.

Instead of considering the model to be a product we consider the act of modeling as a process that entails the development and validation of an entity that accepts descriptions of organizational form, policy and the tangible and intangible factors that determine how the system evolves with time (Forrester 1961). The burden of modeling the problem at hand is thus shifted to the eventual decision-makers and stakeholders. Their direct participation provides them an opportunity to shape the model assumptions and relationships. This increases their willingness to rely on the simulation outcomes. As researchers we have developed a platform that allows them to iteratively change and interact with the model till they are convinced that it is most useful for their purposes. Instead of defining the purpose of the model on 
their behalf and establishing specific choices for them to choose from, we allow them to generate strategies, experiment with outcomes and use the results of their analysis to redefine and streamline their decision-making strategies.

Therefore the significance of this approach is that it couples model development and validation into a single process, while separating the platform/computer software development and verification from it. Hence, project managers who tend to be domain specialists can use the platform to develop strategies and consensus to resolve alternative project risk models, while simulation developers develop verifiable systems. The approach exploits the native strengths of each community while encouraging the easy deployment and use of simulations.

\section{CONCLUSION}

In this paper, we presented an interactive simulation platform called the Interactive Construction Decision Making Aid (ICDMA) and explained the modules in the design and implementation. The platform separates the complexities of developing the model from programming the model and thus encourages the decision-maker's participation into the model development, simulation deployment, experimentation, and validation phases. ICDMA has several additional advantages:

- It provides a basic set of features that allows researchers to quickly implement a construction project based on the as-built plan. Customized features can be utilized using the constraint language provided by the system.

- It can represent constraints between resources and activities. For example, an activity utilizing just-in-time delivery might get delayed and the uninstalled steel members can cause a space violation when the next shipment of steel members arrives. Similarly, the effects of any resource problem on the schedule can be represented. For example, the delivery of wrong material can cause delays in the activities.

- It provides a flexible testbed for algorithms that simulate construction projects.

- Its modular design makes it scalable to larger projects.

Our future work involves improving the expressive power of the underlying languages to support the development of diverse construction project scenarios.

\section{REFERENCES}

Anderson, G. R., A. Mukherjee, and N. Onder. 2009. Traversing and querying constraint driven temporal networks to estimate construction contingencies. Automation in Construction 18 (6): 798-813.

Daccarett, V., and T. Mrozowski. 1999. Aisc digital library: Construction management of steel construction. Available via http://www.aisc.org/content.aspx?id=21250 [accessed 10/26/2009].

Forrester, J. W. 1961. Industrial dynamics. The MIT Press.

Martinez, J. 2009. Methodology for constructing discrete-event simulation studies in construcion engineering and management. Journal of Construction Engineering and Management, Special Issue on Methodology.

Mukherjee, A., N. Onder, and E. Rojas. 2009. Research methods associated with situational simulations in construction. In Proceedings of the Construction Research Congress, April 4th-7th, Seattle, WA., ed. S. T. Ariaratnam and E. Rojas.

OSHA 1996. Construction safety and health outreach program: Steel erection. Available via http://www.osha.gov/[accessed 3/30/2007].

Pritsker, A. A. B. 1992. Simulation: The premier technique of industrial engineering. Industrial Engineering 24 (7): 25-26.

Rojas, E., and A. Mukherjee. 2003. Modeling the construction management process to support situational simulations. Journal of Computing in Civil Engineering 17 (4): 272-280.

Rojas, E., and A. Mukherjee. 2005a. A general purpose situational simulation environment for construction education. Journal of Construction Engineering and Management, ASCE 131 (3): 319-329.

Rojas, E., and A. Mukherjee. 2005b. Interval temporal logic in general purpose situational simulations. Journal of Computing in Civil Engineering 19 (1): 83-93.

Watkins, M., A. Mukherjee, and N. Onder. 2008, December. Using situational simulations to collect and analyze dynamic construction management decision-making data. In WSC '08: Proceedings of the 40th Conference on Winter Simulation, 2377-2386: Winter Simulation Conference, Miami, Florida.

\section{AUTHOR BIOGRAPHIES}

COREY TEBO is a MSc student at Michigan Technological University, with interests in interactive simulation, aesthetic design and practical problem solving. He hopes to work on real-time simulation and video games to enhance learning, education as well as enable creative hypothesis exploration and ease followup on unexpected results. His web page can be found via <http://www. cs.mtu.edu>. 
AMLAN MUKHERJEE is an Assistant Professor at the Department of Civil and Environmental Engineering at Michigan Technological University. His research interests are artificial intelligence technologies such as agent based modeling and temporal logic, construction engineering and management, interactive simulations, construction engineering education, and cognitive modeling of expert decision making. His web page can be found via $\langle$ http: //www. cee. mtu. edu>.

NILUFER ONDER is an Associate Professor at the Department of Computer Science at Michigan Technological University. Her research interests are artificial intelligence, planning, planning under uncertainty, and decision making under uncertainty. Her web page can be found via <http://www. cs.mtu. edu $>$. 